\title{
El crecimiento de la literatura sobre la ley de Bradford
}

\author{
Rubén Urbizagástegui Alvarado *
}

Artículo recibido: 17 de junio de 2013. Artículo aceptado: 9 de marzo de 2015.

\section{Resumen}

Se analiza la literatura producida sobre la ley de Bradford desde 1934 hasta junio del 2012. Se encontraron 936 documentos producidos por 1123 autores diferentes. Estos autores utilizaron 16 idiomas para comunicar los resultados de sus investigaciones; el inglés fue el idioma dominante, seguido del chino, español y portugués. Se identificó también a 15 autores como los más productivos, quienes publican sus investigaciones en inglés, en la forma de artículos en revistas académicas, y las presentan en congresos del área. Esta literatura crece de forma exponencial $\left(R^{2}=0.974\right)$, a una tasa de $5.4 \%$ al año y duplica su tamaño cada 13.2 años.

University of California, Riverside, California, USA. ruben@ucr.edu

INVESTIGACIÓN BIBLIOTECOLÓGICA,Vol.30,Núm.68,enero/abril,2016,México,ISSN: 0187-358X. pp. 51-72 
Palabras clave: Ley de Bradford; Crecimiento de la literatura; Bibliometría; Informetría; Cienciometría.

\begin{abstract}
Growth of Literature on Bradford's Law

Rubén Urbizagástegui-Alvarado
\end{abstract}

This paper analyzes the literature produced on Bradford' Law from 1934 to June 2012. The study compiled 936 documents produced by 1,123 authors, working in sixteen languages to communicate the results of their research, with English the leading language, followed by Chinese, Spanish and Portuguese. The study reveals the fifteen most productive authors, all of which work in English in both academic journals and conference settings. This literature is growing exponentially $\left(R^{2}=0.974\right)$, at a rate of $5.4 \%$ per year and doubling in size every 13.2 years.

Keywords: Bradford's law; Exponential growth; Bibliometrics; Infometrics; Scientometrics.

\title{
Introducción
}

E I término "literatura" se refiere a un conjunto de documentos publicados sobre un asunto determinado. Generalmente son documentos científicos que incluyen una serie de modalidades como artículos de revistas, capítulos de libros, ponencias presentadas en congresos, folletos, monografías, libros, tesis, literatura gris, etc. Este término puede tener diferentes niveles de granularidad, por ejemplo, referirse a una literatura general como "la literatura de química" o del campo de la química, pero también puede especificar documentos más restringidos como la literatura sobre "el efecto invernadero". Sin embargo, lo que se considera conocimiento en un campo científico se extiende más allá de la literatura formal, aunque la mayor parte de ese conocimiento procede de esa literatura; "debemos considerar al conocimiento en un determinado campo consistiendo de tres capas: primero, existen los estudios originales que conducen los investigadores y que luego publican. Después, vienen las revisiones sistemáticas o conceptuales de esos estudios que proporcionan resúmenes y nuevas interpretaciones construidas 
a partir de esa literatura pero extendiéndose más allá de la propia literatura original. Finalmente están las percepciones, conclusiones, e interpretaciones que la gente comparte en conversaciones informales y que se convierten en parte de la tradición del campo" (Kennedy, 2007: 141). Esa literatura publicada es recogida e indizada en bases de datos bibliográficas especializadas, pero conforme crece el conocimiento se incrementa también la literatura que lo explicita. Por lo tanto, es necesario estudiar la forma de crecimiento de esta literatura publicada, poniendo especial atención en su tasa de crecimiento anual y su tasa de duplicación.

La ley de Bradford fue propuesta en 1934. A partir de esa fecha abundante literatura ha sido producida y publicada, pero hasta donde es de conocimiento del autor de este artículo, se han realizado sólo bibliografías (Quemel et al., 1980) y revisiones de la literatura (Lockett, 1989), pero no análisis del crecimiento de la literatura publicada sobre esta técnica bibliométrica. Por esa razón el objetivo de este trabajo es analizar el crecimiento de la literatura publicada sobre la ley de Bradford desde su formulación inicial en 1934 hasta junio del 2012, un largo periodo de 79 años en el que se espera que la literatura publicada se acumule y crezca. Este modelo matemático, junto con otros modelos estadísticos como la ley de Lotka, la ley de Zipf, la regla 80/20, los modelos de crecimiento y obsolescencia de la literatura, el análisis de citas, etc., forman la estructura orgánica del campo de la bibliometría (Urbizagástegui, 2007).

Bradford (1934) postuló como hipótesis que la mayoría de los artículos sobre un asunto especializado podría estar siendo publicada por unas cuantas revistas especialmente dedicadas a ese asunto, en conjunto con ciertas revistas de frontera y muchas otras generales o de dispersión. Por lo tanto, el conjunto de revistas de un campo determinado actuaría como una familia de generaciones sucesivas de parentesco disminuyente donde cada generación sería de mayor número que la generación precedente. La aplicación práctica de la ley de Bradford proporciona los mecanismos para seleccionar las publicaciones periódicas no sólo más productivas sino también más relevantes para cubrir una determinada área del conocimiento. Cuando se estudia el uso de la literatura de un determinado asunto en la forma de citas o uso interno en las bibliotecas y centros de información, también proporciona los mecanismos para no seleccionar (descartar) las revistas y libros poco usados, es decir, el material bibliográfico candidato a almacenamiento en centros regionales de depósitos de baja intensidad de demanda. Es el crecimiento de esta literatura el objeto de estudio del presente artículo. Las preguntas que se busca responder son las siguientes: ¿cuáles son los tipos de documentos publicados sobre este asunto? ¿Cuáles son los idiomas usados para comunicar 
los resultados de las investigaciones? ¿Quiénes son los autores más productivos sobre la ley de Bradford? ¿Qué tipos de documentos producen estos autores y en qué idiomas? ¿La literatura publicada sobre la ley de Bradford está en crecimiento o ya alcanzó su punto de saturación? Y si está creciendo, ¿cuál es la tasa de este crecimiento y cuál es la tasa de duplicación?

Para lograr el objetivo propuesto, este artículo está organizado en seis partes. En la primera se presenta una introducción al tema, se detalla el problema y se formulan las preguntas de investigación. La segunda parte expone el referencial teórico sobre los estudios del crecimiento de la literatura. La tercera parte describe la metodología empleada en la aplicación del modelo de crecimiento escogido con énfasis en la forma de recolección de los datos y la forma de medición de los mismos. En la cuarta parte se exponen los resultados y en la quinta se proponen las conclusiones y discusiones de los resultados obtenidos; por último, se presenta la bibliografía de la literatura revisada.

\section{Referencial teórico}

La base del conocimiento de cualquier campo científico es su literatura publicada, por lo tanto, una medida cuantitativa de su volumen es el tamaño y la estructura de esa literatura, pues cualquier nueva información y contribución florece de las publicaciones anteriores (Braun, Schubert y Kostoff, 2000). Por esa razón, una de las características más obvias de la práctica científica ha sido el crecimiento de la literatura publicada. En esa literatura aparecen nuevos problemas, nuevos métodos para enfocar los problemas de investigación, puntos de vista novedosos o aplicaciones en diferentes líneas de investigación o subcampos del saber.

El crecimiento de la literatura se expresa en la forma de una tasa media estimada por métodos estadísticos aplicados a los datos ordenados cronológicamente según los años de publicación. Las exploraciones iniciales sólo prestaban atención a la forma de crecimiento pero no a la estimación de la tasa de crecimiento. Por ejemplo, Houzeau y Lancaster (1880, citados por Jaschek, 1989: 164) compilaron el número total de artículos escritos sobre astronomía, cubriendo un periodo de 170 años, y mostraron que esa literatura crecía de forma exponencial. Tamiya (1931) estudió la literatura sobre "aspergillus" mostrando que crecía de forma logística; Wilson y Fred (1935) estudiaron la literatura sobre la fijación de nitrógeno por las plantas y verificaron que su crecimiento seguía una curva logística. Para Crane (1944) la literatura de química crecía de manera exponencial, e igualmente la literatura de bioquímica (Schwartz y Powers, 1963) 
Estas exploraciones iniciales alcanzaron consistencia metodológica sólo a partir de 1951, cuando Price (1951) comenzó sus investigaciones sobre el crecimiento de la ciencia objetivada en la literatura científica publicada. Este autor sostuvo que "el número de artículos científicos publicados cada año puede ser tomado como una indicación aproximada de la actividad desplegada en cualquier campo de investigación general o especializada" (Price, 1951: 86). Analizó estadísticamente el Physics Abstracts, que cubre un amplio campo general de la física, y la teoría de los determinantes y matrices, una rama especializada de las matemáticas. Encontró que durante tiempos normales un campo general como la física crece exponencialmente con un alto grado de precisión. Sin embargo, un campo especializado como la teoría de determinantes y matrices crece exponencialmente sólo hasta cierto punto, a partir del cual cambia a un crecimiento lineal. La literatura se duplica cada $10 \mathrm{u} 11$ años en ambos casos.

Más adelante, Price (1956) sostuvo que contándose, por ejemplo, el número de resúmenes del Physics Abstracts y del Chemical Abstracts en cada año, se puede obtener una forma de medida del número de artículos publicados en esos campos durante un determinado periodo. De esos datos se pueden obtener tres conclusiones importantes: casi todas las curvas de crecimiento muestran la misma tendencia; el crecimiento es exponencial, y la constante de la curva exponencial es capaz de efectuar una duplicación de tamaño en un periodo de 10 a 15 años. Aparentemente la ley exponencial gobierna el tamaño de la ciencia. Es más, "los datos que pueden ser llevados a cabo desde el año 1700 o aún antes indican claramente que en general el "tamaño" de la ciencia ha venido creciendo de esta manera en todo el periodo desde la revolución científica y los tiempos de Newton" (Price, 1956: 518). Al año siguiente, Conrad (1957) estudió el crecimiento de la literatura sobre biología y su cobertura por el Biological Abstracts, a partir de lo cual sugirió un crecimiento exponencial de esta literatura y estimó que en 2010 tendríamos una producción de 348000 artículos sobre el asunto. También Strong y Benfey (1960) realizaron un estudio del crecimiento de la literatura dequímica en elChemical Abstractyel Handbuch derOrganischen Chemie de Beilstein con base en el que indicaron que el crecimiento de la información química aproximadamente se duplica cada 13 años.

Posteriormente, Price (1963) estudió el crecimiento de la literatura en el campo de la física contando el número de resúmenes en el Physical Abstracts de 1900 a 1950. Price constató que la literatura de física crecía de forma exponencial con un periodo de duplicación de aproximadamente cada 12 años. May (1966) estudió el crecimiento de la literatura de matemática y usó dos bases de datos: el Jahrbuch über die Fortschritte der Mathematik desde 1868 
hasta 1940, y el Mathematical Reviews de 1941 hasta 1965. Este autor verificó que el número de publicaciones por año había crecido de 800 a 13 000, con una media compuesta de aproximadamente $2.5 \%$ por año y duplicándose aproximadamente cada 28 años, esto es, cuatro veces en un siglo. Stoddart (1967) estudió el crecimiento del número de revistas y de sociedades en el campo de la geografía. Encontró una tasa de crecimiento exponencial de las revistas con un periodo de duplicación cada 30 años; encontró también que las sociedades geográficas crecen de forma exponencial, con una tasa de duplicación de 22 años.

Más tarde, Menard (1971) examinó la literatura en diversos subcampos de la geología; encontró que en paleontología de los vertebrados la literatura producida creció lentamente hasta finales del siglo XVIII y después comenzó a crecer exponencialmente, con un periodo de duplicación de 15 años. Brookes (1973) afirmó que por el número de artículos publicados cada año la literatura producida en la mayoría de los campos científicos tendía a un crecimiento exponencial, con una tasa de duplicación de cada 10 años. A partir de esas épocas se insiste que "en el campo de la ciencia hay un acrecentar acumulativo de las contribuciones que se asemeja a una pared de ladrillos. Cada investigador agrega su ladrillo a la pared en una secuencia ordenada que debe, al menos en teoría, permanecer en perpetuidad como un edificio intelectual construido por habilidades y artificios, descansando sobre los fundamentos primigenios, alargándose hasta los límites superiores del creciente frente de investigación del conocimiento" (Price, 1975: 162). Este autor da ejemplos de como "[...] el número de revistas ha crecido exponencialmente en vez de linealmente. En vez de haber solamente un número exacto de nuevas revistas por año, el número se ha duplicado cada determinado número de años. Realmente la constante es de cerca de 15 años para cada duplicación, correspondiente a un poder de diez en 50 años y a un factor de mil en un siglo y medio" (Price, 1975: 169). Esta "ley exponencial es la consecuencia matemática del tener una cantidad que aumenta de tal modo que cuanto más grande es la cantidad esta crece más rápidamente" (Price, 1975: 169), tanto que "la ley de crecimiento exponencial encontrada para el número de revistas científicas también es obedecida por el número real de artículos científicos en esas revistas" (Price, 1975: 170).

Price es un animador del estudio del crecimiento de las publicaciones, e insistió en que "es remarcable que desde 1918 hasta el presente el número total de artículos de física registrados en los resúmenes [...] ha seguido una curva de crecimiento exponencial en un orden de precisión que no fluctúa más que cerca al $1 \%$ del total. Ahora hay cerca de 180000 artículos de física registrados en esos volúmenes [se refiere al Physics Abstracts] y el número ha 
doblado directamente en una tasa aun más rápida que una vez cada quince años" (Price, 1975: 171). De este tipo de crecimiento se pueden demostrar claramente las siguientes fases: "primero, precursores; después, un constante crecimiento exponencial; a seguir, un decline para un crecimiento linear, cuando no entra en el campo ninguna nueva mano de obra; y finalmente, el colapso del campo, cuando ocasionalmente se producen pocos artículos, o un renacer alternativo, podría repentinamente retomar nueva vida, a través de la redefinición de su contenido y modo de operación" (Price, 1975: 173).

A partir de 1975 y siguiendo las propuestas de Price (1951, 1956, 1963, 1975), se han realizado infinidad de estudios en las diferentes áreas del conocimiento. Por ejemplo, Hall (1989) encontró que para la literatura de geología universal, tomand como base el año 1945, el crecimiento es exponencial con un periodo de duplicación de 8 años, pero el crecimiento de 1945 a 1970 indicó una tasa de duplicación de 6 años. Urbizagástegui y Lane-Urbizagastegui (2008) estudiaron la literatura de plantas medicinales del Perú y descubrieron que esta literatura crece de forma exponencial con una tasa de $6.5 \%$ al año y duplica su tamaño cada 11.3 años. Urbizagástegui (2009) estudió el crecimiento de la literatura publicada sobre la ley de Lotka y encontró que ésta también crece de forma exponencial con una tasa de $7.5 \%$ anual y tiene una tasa de duplicación de 9.6 años. Biglu (2009) estudió la relación entre patentes y publicaciones científicas en el área de medicina indizados en Medline. Todas las publicaciones indizadas con el término "Patentes" en Medline en el periodo de 1965 a 2005 fueron extraídas y analizadas, y muestran un crecimiento exponencial de la literatura con una tasa de crecimiento de $3.1 \%$ anual y una tasa de duplicación de 22.5 años. Nuevamente Urbizagástegui y Lane-Urbizagastegui (2007) analizaron la literatura de plantas usadas como colorantes naturales y encontraron que creció de forma exponencial con una tasa anual del $3.4 \%$ y duplicó su tamaño cada 20.7 años. Finalmente, Restrepo (2011) estudió la literatura producida por los historiadores investigadores de El Colegio de México, la cual adopta la forma exponencial, con una tasa de crecimiento anual del $7.1 \%$ y duplicando su tamaño cada 10.1 años.

\section{Material y métodos}

Como unidades de análisis se tomaron cada uno de los artículos publicados en revistas académicas, capítulos de libros y trabajos presentados en congresos sobre la ley de Bradford o que aplicaran este modelo a alguna disciplina o subcampo determinado. Se excluyeron libros, tesis, monografías y literatura 
gris porque este tipo de publicaciones no son indizadas en las bases de datos bibliográficas consultadas para esta investigación. El periodo cubierto va desde 1934 hasta junio del 2012. Para recolectar los datos se hicieron búsquedas usando los términos "Bradford's law", "Bradford's distribution", "Bradford Scattering law", "Bradford type distribution", "Bradford analysis", "Bradford curve", "Bradford zones" y "Bradford core journals" en sus diferentes acepciones idiomáticas (inglés, francés, alemán, portugués, español, etc.), en los títulos, descriptores y resúmenes de las siguientes bases de datos bibliográficas: Library Literature \& Information Science Full Text, Library and Information Science Abstract (LISA), Library, Information Science \& Technology Abstracts (LISTA), Agrícola, Biosis, CAB Abstracts, Medline, Anthropological Literature, Anthropological Index, Anthropology Plus, WorldCat, HAPI, ArticleFirst, Science Citation Expanded Index, Web of Science, Scopus, Elsevier, JSTOR y otras 120 disponibles en la biblioteca de la Universidad de California en Riverside, incluyéndose bases de datos bibliográficas españolas (ISOC, ICYT y Dialnet). También se consultaron bases de datos latinoamericanas, como INFOBILA de México y LICI del Instituto Brasileiro de Informação em Ciência e Tecnologia (IBICT); bases de datos chinas, como China Academic Journals via EastView Online Services, y japonesas via CiNii: Citation Information by National Institute of Informatics, Japanese Scholarly \& Academic Information, así como bases de datos rusas (Russian Academy of Sciences Bibliographies), alemanas, árabes, etcétera. Igualmente sevisitaron repositorios como Scielo Brasil, Scielo México, Scielo Venezuela, Scielo Colombia, Scielo Chile, Scielo Argentina y Scielo Bolivia, entre otros.

Las referencias identificadas se trasladaron a EndNote X5 para la elaboración de una base de datos específica sobre este asunto. Posteriormente fue realizada una minuciosa lectura de cada uno de los documentos identificados en la búsqueda, dedicando especial atención a cada referencia efectuada en el documento leído. Después, cada referencia relativa a la ley de Bradford era confrontada con la base de datos e incorporada, en caso de no haberse identificado en la búsqueda previa. Las referencias duplicadas fueron eliminadas, manteniendo una sola aparición. Con esta lectura minuciosa se produjo una bibliografía que lista un total de 936 referencias producidas entre 1934 y junio de 2012, entre artículos de revistas, capítulos de libros, comunicaciones presentadas en congresos y otras modalidades de comunicación, la cual constituye el universo de esta investigación. El periodo cubierto por la literatura recuperada es suficientemente extenso como para esperar alguna forma de crecimiento.

Sin embargo, cabe aclarar que sin saber como será la forma de la nube de puntos de los datos del crecimiento de la literatura, es difícil presentar la for- 
ma de medición de los datos recolectados. Es imposible afirmar de antemano si esta literatura tomará una forma linear, exponencial, del poder, Gompertz, etcétera, pero se espera que sea exponencial pues es la forma más común encontrada en los estudios de literaturas que abarcan largos periodos, como en este caso. El crecimiento exponencial representa el aumento de la población en una proporción fija en cada unidad de tiempo; se expresa en porcentajes, con una tasa de crecimiento constante y con un límite de crecimiento no definido. El modelo no solamente proporciona una tasa media de crecimiento, sino también ofrece una tasa de duplicación. Generalmente, el crecimiento exponencial asume una forma cóncava inicial en su representación gráfica. Esta función se representa matemáticamente como sigue:

$$
C(t)=C O e a^{t}
$$

Siguiendo a Egghe \& Ravichandra Rao (1992), esta función puede ser reescrita como:

$$
\begin{aligned}
& C(t)=c g^{t} \\
& \text { donde } \\
& c>0, g>1, y t \geq 0 .
\end{aligned}
$$

Cuando se estudia el crecimiento de la literatura se está postulando una relación entre el tiempo (variable independiente), medido en años, y el volumen acumulado de la literatura producida (variable dependiente), medida en unidades producidas. Se supone que esa relación bivariante puede ser estadísticamente modelable. Para evaluar el ajuste del modelo se construye una nube de puntos dispersos que están basados en los datos observados. Eso permite evaluar si existe cierta regularidad en la distribución de las frecuencias observadas y, cuando esa regularidad se asemeja a la curva mostrada en el gráfico, se intenta ajustar esa curva a la nube de puntos, vía la regresión no-linear. En una distribución exponencial lo que se va probar es si el volumen acumulado de la producción de documentos según los años t procede de una distribución exponencial, esto es, la probabilidad de que una frecuencia en la muestra sea igualmente probable para todas las frecuencias en la misma situación.

El cálculo de los parámetros de la distribución exponencial se realizó por el método de la determinación de la regresión de la curva no-linear, usando el software estadístico SPSS 17.0 para Windows. Como se espera una alta corre- 
lación entre las variables dependientes e independientes, esa correlación fue explorada con el coeficiente de determinación $\mathrm{R}^{2}$ al 0.01 nivel de significancia.

\section{Resultados}

Se encontraron 936 publicaciones producidas por 1123 autores diferentes. La Tabla 2 muestra la cantidad de documentos publicados sobre la ley de Bradford desde 1934, cuando fue inicialmente propuesta por Bradford (1934), hasta junio del 2012. El volumen de documentos fue dividido y organizado por décadas.

Tabla 2. Cantidad de documentos publicados según las décadas

\begin{tabular}{|c|c|c|}
\hline Décadas & Núm.dedocumentospublicados & Porcentaje \\
\hline $2003-2012$ & 333 & 35.58 \\
\hline $1993-2002$ & 190 & 20.30 \\
\hline $1983-1992$ & 174 & 18.59 \\
\hline $1973-1982$ & 174 & 18.59 \\
\hline $1963-1972$ & 54 & 5.77 \\
\hline $1953-1962$ & 5 & 0.53 \\
\hline $1943-1952$ & 4 & 0.43 \\
\hline $1934-1942$ & 2 & 0.21 \\
\hline Total & 936 & 100.0 \\
\hline
\end{tabular}

La literatura publicada sobre este asunto ha estado en permanente crecimiento década a década y ha pasado de representar sólo $0.2 \%$ en la primera década (1934-1942) hasta alcanzar $19 \%$ en la quinta década (1973-1982), para luego constituir $36 \%$ del total de la literatura publicada en la última década (2003-2012). El rango de la distribución va de 1 documento en el nivel más bajo hasta 52 publicaciones en el nivel más elevado. La media de documentos publicados es de $11.85 \pm 12$ documentos por año, con una desviación estándar de 1.4 documentos; la mediana es de 13 documentos, y una varianza de 155.6 documentos publicados con una desviación estándar de 12.5 publicaciones.

La Figura 1 muestra este crecimiento según las décadas en la forma de un gráfico de barras. A partir de la década de 1934 a 1942, la literatura publicada va creciendo gradualmente hasta alcanzar su mayor expresión en volumen en la última década (2003-2012). La gradación creciente es notable a simple vista y no precisa mayores explicaciones. Los años expresados en las décadas significan que van desde el año siguiente hasta el año indicado en el gráfico. Por ejemplo, 1972 significa que la década va desde 1963 hasta 1972. 


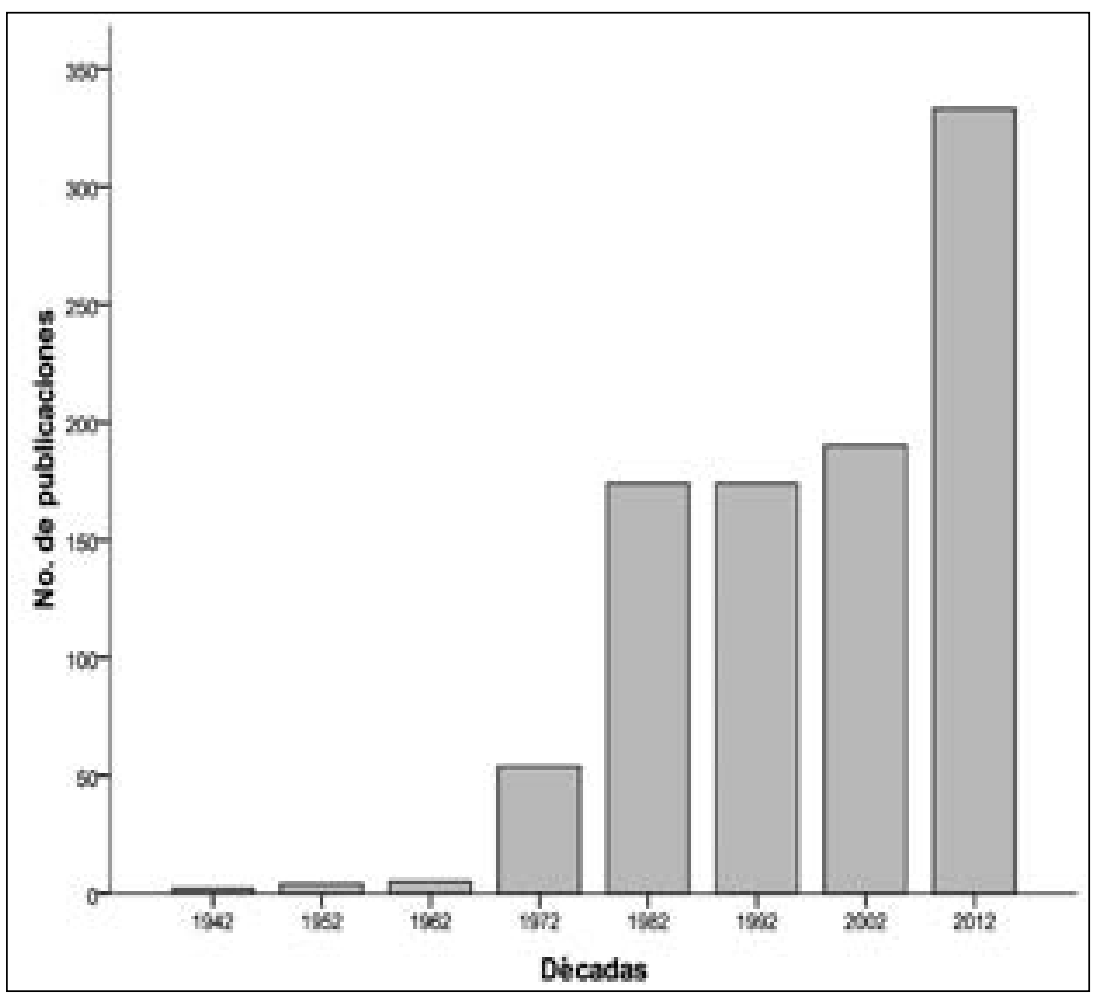

Figura 1. Volumen de publicación según las décadas

Los 1123 autores identificados en esta investigación utilizaron 16 idiomas diferentes para publicar los resultados de sus investigaciones sobre la ley de Bradford. La Tabla 3 muestra que las publicaciones en inglés alcanzaron un $65 \%$ de las contribuciones y dejaron apenas un espacio de $35 \%$ para ser compartido por los otros idiomas. En ese aspecto, las publicaciones en chino ocupan el segundo estrato, con $12 \%$ de las contribuciones, seguidas del español, con $9 \%$, el portugués con $6 \%$, el ruso con $2 \%$, el japonés con $1 \%$, el alemán con $1 \%$, el francés con $0.8 \%$, el árabe con $0.6 \%$, el rumano con $0.6 \%$, el turco con $0.4 \%$, el danés con $0.3 \%$, el eslovaco con $0.3 \%$ y, finalmente, el checo y el italiano con apenas $0.1 \%$ de los trabajos publicados. Esto no es nada sorprendente ya que es conocida la hegemonía del idioma inglés en la comunicación científica, tanto así que Price (1971) afirmaba que en este idioma se publica poco más de la mitad de la producción filosófica y científica del mundo. 
Tabla 3. Tipos de documentos publicados según los idiomas

\begin{tabular}{|l|c|c|c|c|c|c|c|}
\hline Idiomas & $\begin{array}{c}\text { Capítulos } \\
\text { de libros }\end{array}$ & $\begin{array}{c}\text { Artículos } \\
\text { revistas }\end{array}$ & $\begin{array}{c}\text { Ponencias } \\
\text { congresos }\end{array}$ & $\begin{array}{c}\text { Artículos } \\
\text { electrónicos }\end{array}$ & Informes & $\begin{array}{c}\text { Cartas } \\
\text { al editor }\end{array}$ & Total \\
\hline Alemán & - & 8 & 1 & - & - & - & 9 \\
\hline Árabe & - & 6 & - & - & - & - & 6 \\
\hline Checo & - & 1 & - & - & - & - & 1 \\
\hline Chino & - & 116 & - & - & - & - & 116 \\
\hline Danés & 1 & 2 & - & - & - & - & 3 \\
\hline Eslovaco & 1 & 1 & 1 & - & - & - & 3 \\
\hline Español & 1 & 70 & 11 & 1 & - & - & 83 \\
\hline Francés & 2 & 6 & - & - & - & - & 8 \\
\hline Húngaro & - & 2 & - & - & - & - & 2 \\
\hline Inglés & 15 & 514 & 61 & 1 & 2 & 18 & 611 \\
\hline Italiano & - & 1 & - & - & - & - & 1 \\
\hline Japonés & - & 11 & - & - & - & - & 11 \\
\hline Portugués & - & 42 & 11 & - & - & - & 53 \\
\hline Rumano & - & 6 & - & - & - & - & 6 \\
\hline Ruso & - & 19 & - & - & - & - & 19 \\
\hline Turco & - & 4 & - & - & - & - & 4 \\
\hline Total & 20 & 809 & 85 & 2 & 2 & 18 & 936 \\
\hline
\end{tabular}

La forma más popular de publicación es el artículo de revista con el $86 \%$ del total de publicaciones en todos los idiomas; siguen las ponencias presentadas en congresos (9\%) y los capítulos de libros (2\%). Estas tres formas representan el $98 \%$ de todo lo publicado hasta junio del 2012. En inglés se publican todos los tipos de escritos, con alta concentración en artículos de revistas académicas ( $84 \%$ de todo lo publicado en este idioma), pero con menos énfasis en ponencias presentadas en congresos (10\%) y capítulos de libros (2.5\%). En español y portugués el comportamiento es casi similar. En los otros idiomas la publicación se da preferentemente como artículos de revistas. Es curioso notar que las cartas al editor no aparecen en los idiomas diferentes al inglés identificados en esta investigación.

La Tabla 4 muestra a los autores más productivos organizados con base en los idiomas en los cuales publican sus investigaciones. La mayoría de estos autores publican en inglés. De los 15 autores más productivos, Ming-Yueh Tsay publica también en chino, su idioma nativo. Francisco López Muñoz, a pesar de ser español, se esfuerza en publicar también en inglés, seguramente con la idea de "alcanzar una visibilidad mas justa" (Miranda, 1982, 1998) de su producción científica. Yasar Tonta, de Turquía, prefiere publicar en inglés al igual que Philipp Mayr, de Alemania. Urbizagástegui, en cambio, publica en portugués y en español como una forma de reproducir su cultura y resistir a la anglización de la investigación en ciencia y tecnología. Todos estos auto- 
res publican mayoritariamente artículos en revistas académicas y presentan los resultados de sus investigaciones en forma de ponencias en congresos especializados del área.

Tabla 4. Autores más productivos según los idiomas en que publican

\begin{tabular}{|l|c|c|c|c|c|c|c|c|}
\hline & \multicolumn{9}{|c|}{ Idiomas } \\
\hline Autores & Inglés & Chino & Francés & Español & Portugués & Turco & Alemán & Total \\
\hline Brookes, Bertram C. & 26 & - & - & - & - & - & - & 26 \\
\hline Tsay, Ming-Yueh & 8 & 5 & - & - & - & - & - & 13 \\
\hline Urbizagástegui,Rubén & - & - & - & 6 & 6 & - & - & 12 \\
\hline Rousseau, Ronald & 12 & - & - & - & - & - & - & 12 \\
\hline Egghe, Leo & 11 & - & 1 & - & - & - & - & 12 \\
\hline Leimkuhler, F. F. & 8 & - & - & - & - & - & - & 8 \\
\hline LópezMuñóz,Francisco & 5 & - & - & 2 & - & - & - & 7 \\
\hline Oluic-Vukovic, Vesna & 7 & - & - & - & - & - & - & 7 \\
\hline Bradford, Samuel C. & 6 & - & - & - & - & - & - & 6 \\
\hline Wilson,ConcepcionS. & 6 & - & - & - & - & - & - & 6 \\
\hline Bookstein, Abraham & 6 & - & - & - & - & - & - & 6 \\
\hline Sen, Subir K. & 6 & - & - & - & - & - & - & 6 \\
\hline Tonta, Yasar & 5 & - & - & - & - & 1 & - & 6 \\
\hline Patra, Swapan Kumar & 6 & - & - & - & - & - & - & 6 \\
\hline Mayr, Philipp & 5 & - & - & - & - & - & 1 & 6 \\
\hline Total & 117 & 5 & 1 & 8 & 6 & 1 & 1 & 139 \\
\hline
\end{tabular}

Apenas un autor (Brookes) envió 6 cartas al editor de varias revistas académicas discutiendo diferentes aspectos de la ley de Bradford. El mismo Brookes fue uno de los grandes animadores de la bibliometría y en especial de la ley de Bradford, sobre la que publicó 20 artículos académicos e hizo una presentación en un congreso del área (ver Tabla 5).

Tabla 5. Tipos de publicaciones de los autores más productivos

\begin{tabular}{|l|c|c|c|c|c|}
\hline \multicolumn{1}{|c|}{ Autores } & Artículos & $\begin{array}{c}\text { Ponencias } \\
\text { congresos }\end{array}$ & $\begin{array}{c}\text { Capítulos } \\
\text { de libros }\end{array}$ & Cartasaleditor & Total \\
\hline Brookes, Bertram C. & 20 & 1 & - & 5 & 26 \\
\hline Tsay, Ming-Yueh & 13 & - & - & - & 13 \\
\hline Urbizagástegui, Rubén & 10 & 2 & - & - & 12 \\
\hline Rousseau, Ronald & 12 & - & - & - & 12 \\
\hline Egghe, Leo & 10 & 1 & 1 & - & 12 \\
\hline Leimkuhler,FerdinandF. & 8 & - & - & - & 8 \\
\hline LópezMuñ́z,Francisco & 6 & - & 1 & - & 7 \\
\hline Oluic-Vukovic, Vesna & 6 & - & 1 & - & 7 \\
\hline BradfordSamuelClement & 2 & 3 & 1 & - & 6 \\
\hline Wilson, Concepcion S. & 4 & 1 & 1 & - & 6 \\
\hline
\end{tabular}




\begin{tabular}{|l|c|c|c|c|c|}
\hline Bookstein, Abraham & 5 & 1 & - & - & 6 \\
\hline Sen, Subir K. & 6 & - & - & - & 6 \\
\hline Tonta, Yasar & 4 & 2 & - & - & 6 \\
\hline Patra, Swapan Kumar & 6 & - & - & - & 6 \\
\hline Mayr, Philipp & 5 & 1 & - & - & 6 \\
\hline Total & 117 & 12 & 5 & 5 & 139 \\
\hline
\end{tabular}

La forma de crecimiento de la literatura producida sobre la ley de Bradford se muestra en la Figura 2. De la forma cóncava inicial, la nube de puntos aumenta constantemente hasta el año 2012, con pequeñas oscilaciones entre $1979-1980$ y $1985-2008$. Esta forma del trazado indica que estamos frente a un crecimiento exponencial. Puede observarse que las publicaciones sobre la ley de Bradford hasta 1968 muestran una condición estable. A partir de esa fecha comienzan a crecer constantemente.

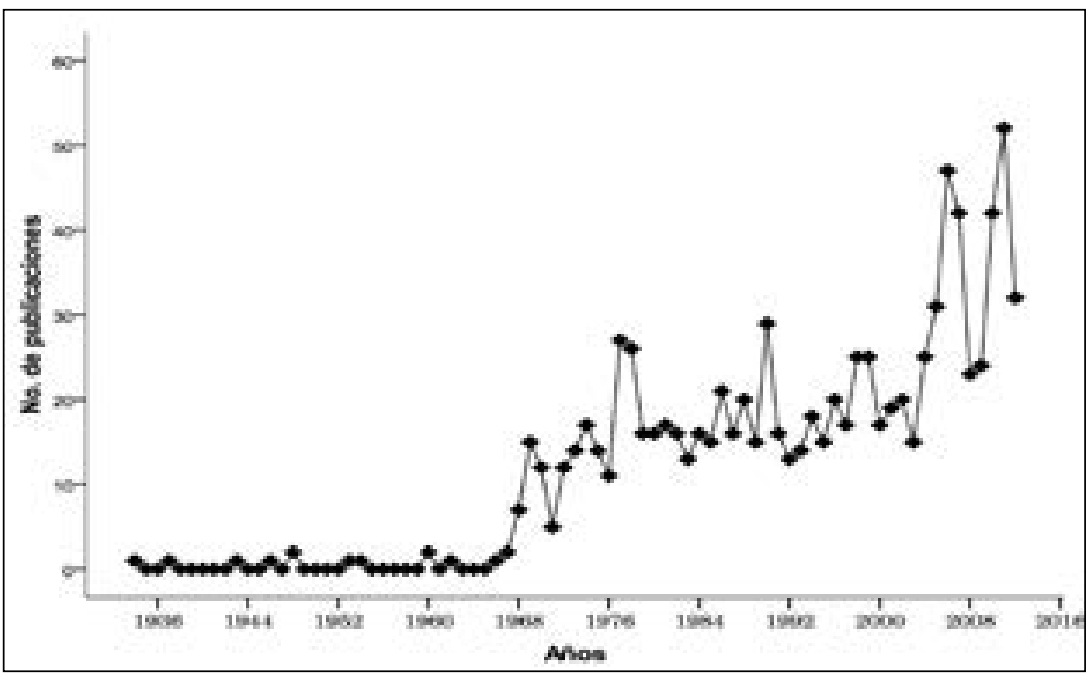

Figura 2. Crecimiento de la literatura según los años, 1934-2012

Desde la formulación inicial de Bradford (1934) no aparece ninguna otra publicación hasta que el propio autor presenta su modelo de la dispersión de artículos en revistas académicas en la 14a Conferencia de ASLIB (Bradford, 1937). Sin tener mucha repercusión, publica artículos en el Proceedings of the British Society for International Bibliography (Bradford, 1943, 1946) e incluye un capítulo titulado "El caos documental" en su libro Documentation (Bradford, 1948). Poco después, es Vickery (1948) quien produce un artículo criticando su formulación matemática. Cinco años después Stevens (1953) 
publica un artículo citando a Vickery (1948) y a Bradford (1948) para explicar las características de dispersión de las literaturas especializadas en un determinado asunto. Es hasta el inicio de la década de 1960 que aparecerán tres referencias al asunto: Fourmont y Kervégant (1960), Kendall (1960) y Cole (1962). A partir de 1966 y hasta 2012 cada año se publican documentos usando las estrategias propuestas por Bradford $(1934,1948)$ para la selección de revistas especializadas en un determinado asunto, por lo menos en una media de 12 artículos utilizando la ley de Bradford. Debemos recordar que en la década de 1960 se publican Science Since Babylon (Price, 1961) y Little Science, Big Science (Price, 1963), que llaman la atención por las posibilidades que ofrece el análisis de la literatura publicada en el proceso de desarrollo de la ciencia y sientan las bases de la bibliometría y la ciencia de la ciencia. En ambos libros se comenta sobre el crecimiento de la literatura y los científicos que trabajan especialmente en la física y la química. Al final de esa década, Pritchard (1969) publica un artículo que introdujo el término "bibliometría". A partir de esa fecha la bibliometría comienza a ser considerada una disciplina de la ciencia de la información y la literatura publicada sobre el asunto comienza a crecer incesantemente. La ley de Bradford se transforma así en uno de los animadores de la práctica bibliométrica. La Figura 4 muestra la distribución de los datos acumulados según los años.

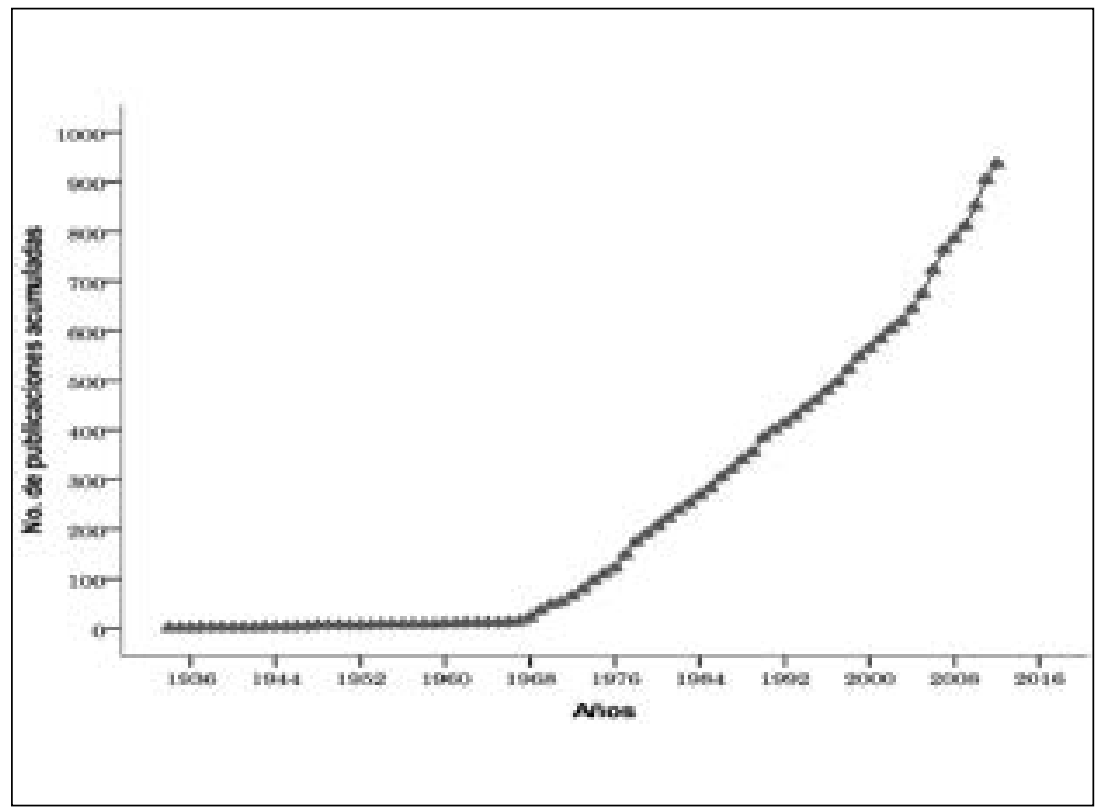

Figura 4. Crecimiento de la literatura acumulada según los años, 1934-2012 
Estos datos acumulados se utilizaron para estimar la tasa de crecimiento y duplicación de la literatura producida sobre la ley de Bradford. Esta literatura se mantiene estable hasta 1968 y a partir de ese año comienza a crecer aceleradamente, formando un trazado creciente casi recto hasta junio del 2012. Por la forma de dispersión de la nube de puntos es evidente que estamos frente a una forma de crecimiento exponencial. La línea casi recta indica que sigue en crecimiento pues no muestra evidencias de haber alcanzado un nivel de saturación. Como lo afirma Price (1975), una literatura puede ser linear, luego transformarse en exponencial para alcanzar su punto de saturación con una forma logística. Este no parece ser el caso de la literatura sobre la ley de Bradford, que pasados 79 años desde su formulación inicial aún sigue en permanente crecimiento.

La Tabla 6 muestra los parámetros y valores obtenidos de la aplicación del modelo exponencial por el método de la regresión no-lineal.

Tabla 6. Valores de los parámetros obtenidos con el modelo exponencial

\begin{tabular}{|c|c|c|c|c|}
\hline \multicolumn{2}{|c|}{} & \multicolumn{2}{c|}{ Intervalo de confianza del 95\% } \\
\hline Parámetros & Valoresestimados & Desvío padrón & Límite menor & Límite mayor \\
\hline $\mathrm{c}$ & 16.835 & 1.704 & 13.354 & 20.316 \\
\hline $\mathrm{g}$ & 1.054 & 1.002 & 1.051 & 1.057 \\
\hline
\end{tabular}

El valor de c fue igual a 16.835 y el de g igual a 1.054. Con esos valores conocidos se puede establecer la siguiente ecuación y así predecir el crecimiento exponencial de la literatura publicada sobre la ley de Bradford:

$$
C(t)=16.835 \times 1.054^{t}
$$

Esta ecuación indica que la literatura sobre la ley de Bradford publicada a partir de 1934 crece a una tasa de $5.4 \%$ al año y que duplica su tamaño cada 13.2 años. Una representación gráfica de los valores observados y de los datos estimados se muestra en la Figura 5 (p. sig.). Obsérvese cuán cerca crecen los valores observados y los estimados por el método de la regresión no-lineal, obteniéndose un $\mathrm{R}^{2}$ igual a 0.974 , que indica que apenas hay una posibilidad de $2.6 \%$ de error en el estimado de los valores esperados. 


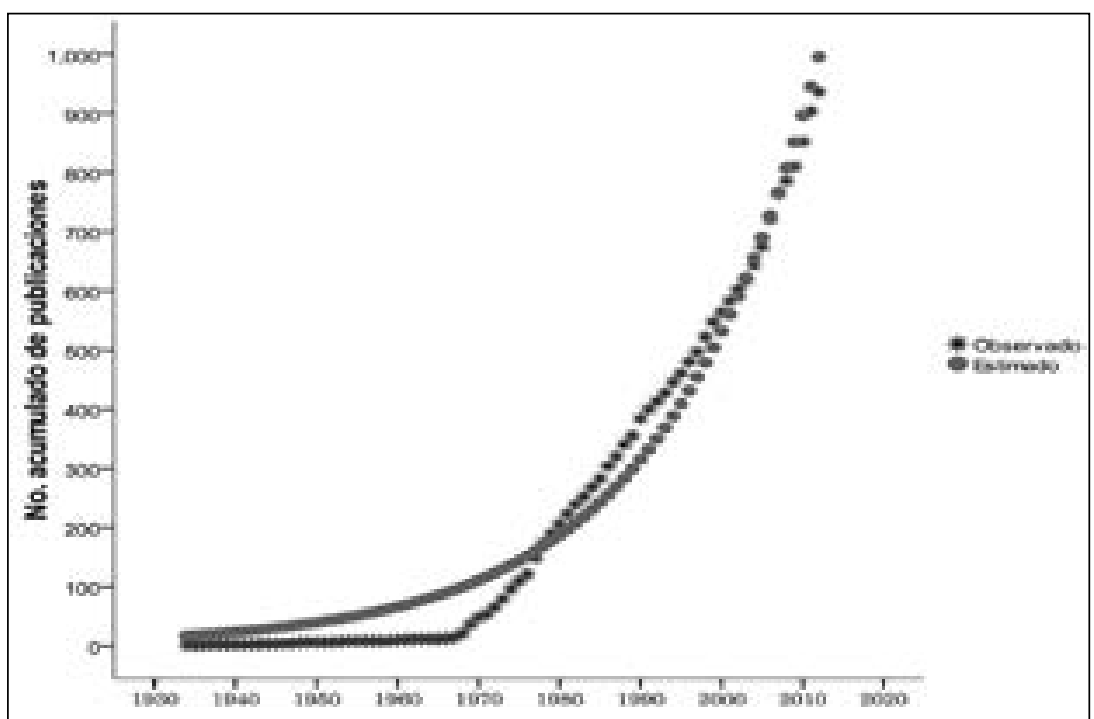

Figura 5. Representación gráfica de los valores observados y estimados

\section{Discusión y conclusiones}

El estudio del crecimiento de la literatura es importante porque compromete la preservación de espacio en las bibliotecas: espacio en los estantes, espacio en la memoria de las computadoras, etcétera. Espacio significa recursos financieros, costos y capital. El crecimiento de la literatura también tiene implicaciones sociológicas ya que envuelve problemas potenciales de acceso para su uso (Egghe, 1994). Su estudio es importante también para predecir su desarrollo futuro. "Una forma muy sencilla de comparar las diversas tasas de crecimiento exponencial es por medio del tiempo de duplicación" (Braun, Lyon y Bujdosó, 1977: 682A) y la tasa de crecimiento anual. Es usual también encontrar la "tasa de crecimiento de una función exponencial descrita por el periodo de duplicación, por el porcentaje de crecimiento anual, o por el índice de exponencialidad" (Gilbert y Woolgar, 1974: 280).

Durante el periodo investigado se observó que el crecimiento de la literatura publicada sobre la ley de Bradford se ajusta al modelo exponencial $\left(\mathrm{R}^{2}=\right.$ 0.974), con una tasa de crecimiento anual de $5.4 \%$ al año y duplica su tamaño cada 13.2 años. La tasa de crecimiento anual está muy cerca al $5.5 \%$ observado por Holt (1968) en el campo de la economía y al $6.5 \%$ anual observado por Urbizagástegui \& Lane-Urbizagastegui (2008) para el crecimiento de las plantas medicinales del Perú. La tasa de duplicación de esta literatura 
está un poco por encima de los 10 años observados por Brookes (1973) para la literatura de las ciencias en general, y ligeramente por debajo de los 15 años observados por Menard (1971) en el campo de la geología. También está dentro del rango de las expectativas de 11 a 15 años observadas por Price $(1951,1956)$ para la física y la química.

Estos resultados contradicen las afirmaciones de Egghe y Ravichandra Rao (1992), en el sentido de que la tasa de duplicación en las ciencias sociales es menor que en las ciencias puras y aplicadas. Si este fuera el caso, la bibliometría, en particular la subdisciplina de la ley de Bradford, estaría más cercana a las ciencias puras y aplicadas que a las ciencias sociales o humanidades. Parece que la tasa de crecimiento de la literatura es independiente de los campos, sean ciencias puras o sociales; más bien son el resultado de la intensidad de la investigación y el volumen de los investigadores operando en esas áreas. Cuanto mayor el número de investigadores trabajando en un determinado campo, mayor las posibilidades de producir más literatura y viceversa. Si en un determinado campo existen 10000 investigadores y cada uno de ellos publica un artículo por año, se agregan 10000 publicaciones anuales a las existentes anteriormente. Pero si existen sólo 100 investigadores que publican un artículo por año, se agregarán solamente 100 artículos a los previamente existentes. Por lo tanto, la tasa de duplicación de los 10000 investigadores será más rápida que de los 100 del otro campo. Esta característica fue observada por Menard (1971) en el campo de la geología, con base en el hecho de que varias subdisciplinas crecían a diferentes tasas y que para una mejor predicción había que prestar atención a las relaciones entre ellas.

En esta investigación se identificaron también 1123 autores que utilizaron 16 idiomas para comunicar sus resultados. Las publicaciones en inglés alcanzaron un $65 \%$ de las contribuciones y dejaron apenas un espacio de $35 \%$ para ser compartido por los idiomas restantes. Nuevamente se verificó la hegemonía del idioma inglés en las comunicaciones del campo de la bibliometría. Sin embargo, hay que tener presente que "el inglés tiene la capacidad de pautar las ciencias sociales, de la misma manera que la portada de un periódico pauta las noticias. Pautar significa dar visibilidad a un tema, pero también ocultar otros" (Ortiz, 2009: s. pág.). Se identificaron 15 autores como los más productivos en este asunto; la mayoría de estos autores publica exclusivamente en inglés. 


\section{Bibliografía}

Biglu, Mohammad Hossein (2009), "Patent literature trends in Medline throughout 1965-2005", en ACIMED, agosto, 20 (1): 1-8.

Bradford S. C. (1934), "Sources of information on specific subjects", en Engineering, 137 (3550): 85-86. [Reimpreso en Journal Information Science, 1985, 10: 176-180]

(1937), "The extent to which scientific and technical literature is covered by present indexing and abstracting periodicals", en Proceedings of 14th ASLLB Conference, London: ASLIB, 59-71.

(1943), "Note on the Scattering of Papers on Specific Subjects In Scientific Periodicals", en Proceedings of the British Society for International Bibliography, 5 (3): 74-75.

(1946), "The Problem of Complete Documentation in Science and Technology", en Proceedings of the British Society for International Bibliography, 8 (3): 39-55.

(1948), Documentation, London: Crosby Lockwood.

Braun, Tibor; Lyon, W. S. \& Bujdosó, E. (1977), "Literature growth and decay: an activation analysis résumé", en Analytical Chemistry, julio, 49 (8): 682A-688A.

; Schubert, András P. \& Kostoff, Ronald N. (2000), "Growth and trends of fullerene research as reflected in its journal literatura", en Chemical Reviews, 100 (1): 23-37.

Brookes, Bertram C. (1973), "Numerical methods of bibliographical analysis", en Library Trends, julio, 22 (1): 18-43.

Cole, P. F. (1962), "A new look at reference scattering", en Journal of Documentation, junio, 18 (2): 58-64.

Conrad, G. Miles (1957), "Growth of biological literature and the future of Biological Abstracts", en Federation Proceedings, julio, 16 (2): 711-715.

Crane, E. J. (1944), "Growth of chemical literature: contributions of certain nations and the effects of war", en Chemical and Engineering News, septiembre, 22 (17): 1478-1481; 1496.

Egghe, Leo y Ravichandra Rao, I. K. (1992), "Classification of growth models based on growth rates and its applications", Scientometrics, 25 (1): 5-46.

Egghe, Leo (1994), "A theory of continuous rates and applications to the theory of growth and obsolescence rates", en Information Process and Management, 30 (2): 279-292.

Fourmont, Raymond y Kervégant, Desire (1960), "Bibliographies et documentation: insuffisances, problemes et perspectives (fin)", Bulletin des Bibliotheques de France, 6: 157-168.

Gilbert, G. Nigel y Woolgar, Steve (1974), "The quantitative study of science: an examination of the literatura", en Science Studies, 4: 279-294.

Hall, D. H. (1989), "Rate of growth of literature in geoscience from computerized databases", en Scientometrics, 17 (1/2): 15-38. 
Holt, Charles C. y Schrank, William E. (1968), "Growth of the professional literature in economics and other fields, and Some Implications", en American Documentation, enero, 19 (1): 18-26.

Houzeau, Jean-Charles y Lancaster, A. (1882-1889), Bibliographie generale de l'astronomie, Bruxelles: F. Hayez.

Jaschek, Carlos (1989), Data astronomy, Cambridge, New York: Cambridge University Press.

Kendall, M. G. (1960), "The bibliography of operational research", en Operational Research Quaterly, mayo-junio, 11 (1/2):31-36.

Kennedy, Mary M. (2007), "Defining a literatura", en Educational Research, abril, 36 (3): 139-147.

Lockett, Mary W. (1989), "The Bradford distribution: a review of the literature, 1934-1987", en Library \& Information Science Research, enero-marzo, 11 (1): 21-36.

May, Kenneth O. (1966), "Quantitative growth of the mathematical literatura", en Science, New Series, diciembre, 154 (3757): 16721673.

Menard, H. W. (1971), Science: growth and change, Cambridge, Mass.: Harvard University Press.

Miranda, Antonio Lisboa Carvalho de (1982), "Latin American periodicals in the field of library Science: an analysis", en 48a. Conferencia General de la IFLA (Montreal, 1982), Río de Janeiro.

(1998), "Produção científica na ciência da Informação", en Ciência da Informação, 27 (1): 308-313.

Ortiz, Renato (2009), "Los intelectuales: No existe ni existirá nunca una sociedad mundial integrada", entrevista publicada en La Nación (Argentina), 2 de septiembre.

Price, John Derek de Solla (1951), "Quantitative measures of the development of science", en Archives Internationales d'Histoire des Sciences, 14 (Quatrieme Annee): 86-93.

(1956), "The exponential curve of science", en Discovery, 17: 240-243. [Reimpreso en Bernard Barber \& Walter Hirsch (ed.), The sociology of science. New York: The Free Press of Glencoe, 1962, 516-524.]

(1961), Science Since Babylon, New Haven, Connecticut: Yale University Press.

(1963), Little Science, Big Science, New York: Columbia University Press.

(1969), "The structures of publication in science and technology", en Willaim H. Gruber \& Donald G. Marquis, Factors in the transfer of technology, 91-104. Cambridge, Mass.: The MIT Press.

(1971), "The expansion of scientific knowledge", en Annals of the New York Academy of Sciences, 184: 257-259.

_ (1975), “Diseases of science”, en Science Since Babylon, ed. ampliada, New Haven y London: Yale University Press, 161-195.

Pritchard, Alan (1969), "Statistical bibliography or bibliometrics?", en Journal of Documentation, diciembre, 25 (4): 348-349. 
Quemel, Maria Angélica Rodrigues; Psquarelli, Maria Luiza Rigo; Carvalho, Neide de y Pereira, Rosa Edite Lemos Alves (1980), "Lei de Bradford: levantamento bibliográfico", en Revista Brasileira de Biblioteconomia e Documentação, julio-diciembre, 13 (3/4): 256-265.

Restrepo Arango, Cristina (2011), "Producción bibliográfica de los historiadores de El Colegio de México", en Investigación Bibliotecológica, mayo-agosto, 25 (54): 111-140.

Schwartz, Ellen Shirley E. y Powers, Wendell H. (1963), "Survey of the quantity and distribution of Biochemical literatura", en Journal of Chemical Documentation, enero, 3: 37-42.

Stevens, Rolland Elwell (1953), Characteristics of subject literatures, Chicago: Publications Committee of the Association of College and Reference Libraries, 10-21. (ACRL monographs, 6)

Stoddart, D. R. (1967), "Growth and structure of geography", en Institute of British Geographers Transactions and Papers, junio, 41: 1-19.

Strong, Laurence E. y Benfey, O. Theodor (1960), "Is Chemical information growing exponentially?", en Journal of Chemical Education, 37 (1): 29-30.

Tamiya, Hiroshi (1931), "Eine mathematische Betrachtung über die Zahlenverhältnisse der in der Bibliographie von Aspergillus zusammengestellten Publikationen", en The Botanical Magazine, 45 (530): 62-71.

Urbizagástegui Alvarado, Rubén y Lane-Urbizagastegui, Shelley (2007), "El crecimiento de la literatura sobre plantas usadas como colorantes naturales y la productividad de sus autores", en Revista AIBDA, enero-junio, 28 (1): 69-110.

_ y Lane-Urbizagástegui, Shelley (2008), "El crecimiento de la literatura sobre plantas medicinales del Perú", en Revista AIBDA, 29 (1/2). Disponible en: http://132.248.9.34/hevila/RevistaAIBDA/ 2008/vol29/no1-2/4.pdf

Urbizagástegui Alvarado, Rubén (2010), “A Cienciometria como um campo científico", en Informação \& Sociedade: Estudos, João Pessoa, septiembre-diciembre, 20 (3): 41-62.

(2009), "Crescimento da literatura e dos autores sobre a Lei de Lotka", en Ciência da Informação, septiembre-diciembre, 38 (3): 111-129.

(2007), "A bibliometria: historia, legitimação e estrutura", en Lídia Maria Batista Brandão Toutain (coord.), Para entender a ciência da informação, Salvador, Bahía, Brasil: EDUFBA, 185-217. (Saladeaula, 5)

Vickery, B. C. (1948), "Bradford's Law of Scattering", en Journal of Documentation, diciembre, 3(4): 198-203.

Wilson, P. W. y Fred, E. B. (1935), "The growth curve of a scientific literature: nitrogen fixation by Plants", en The Scientific Monthly, septiembre, 41 (3): 240-250. 


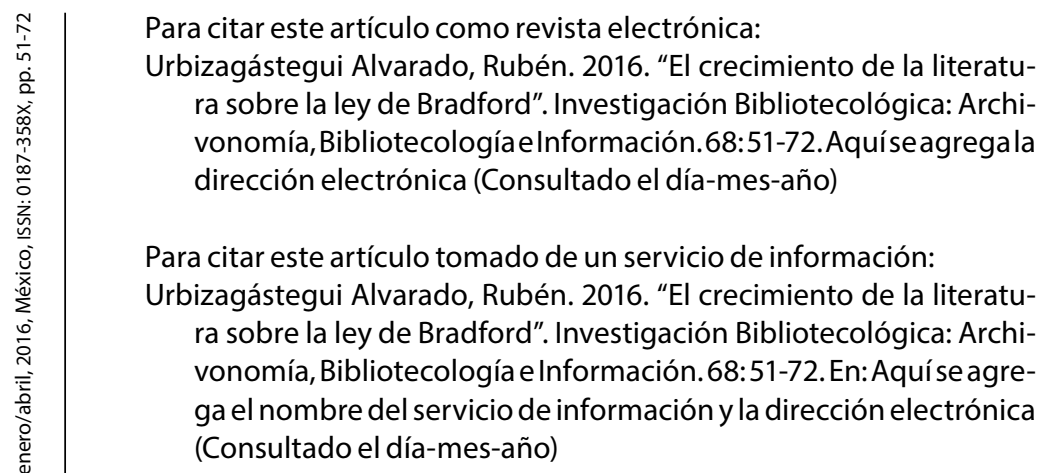

72 